\title{
ON THE LOCAL INTERACTION OF MONEY AND CREDIT ${ }^{1}$
}

\author{
Yi Jin \\ Department of Economics, University of Kansas \\ Lawrence, KS 66045 \\ E-mail: yjin@ku.edu \\ Ted Temzelides \\ Department of Economics, University of Pittsburgh \\ Pittsburgh, PA 15260 \\ E-mail: tedt@pitt.edu
}

JEL: E4, E5

Keywords: Money and Credit, Local Matching

\begin{abstract}
We study the coexistence of monetary and credit transactions in a model where exchange is decentralized. Agents belong to different locations which are informationally separated. The equilibrium mix of monetary and credit transactions is characterized as a function of the frequency of meetings among agents from different locations. Credit transactions take place only among a small set of "neighbors." Monetary trades emerge only if interactions with faraway locations are sufficiently frequent. Even in that case, trades among nearby locations remain non-monetized.
\end{abstract}

\footnotetext{
${ }^{1}$ We thank, without implicating in any way, Dean Corbae, Narayana Kocherlakota, B. Ravikumar, and Steve Williamson for comments and discussions. We also thank an associate editor and a referee for providing comments that greatly improved exposition.
} 


\section{INTRODUCTION}

Transactions between people that meet frequently often involve neither a double coincidence of wants nor the use of money but, rather, some form of "credit." For example, one person might be allowed to borrow some sugar from a next-door neighbor. Instead of offering money, she implicitly agrees to help out a neighbor on a similar occasion in the future. On the other hand, such favors might not be extended to people that we meet only once. In this case, some form of payment, say money, is offered on the spot in exchange for the good or service provided. Of course, people that we meet often do not have to be located literally close to us. For example, people that share certain interests or professional activities might meet frequently with each other regardless of their own geographic location.

We study the coexistence of monetary and credit transactions in a model where exchange is decentralized. We build a model based on the work of Kiyotaki and Wright (1989). The decentralized way in which goods and information are exchanged in this model makes it especially appropriate for the study of issues concerning early economies in which centralized markets were scarce or non-existent. We add two features to the standard setup. First, matching is not uniform but local. Each agent belongs to a certain location (a village). People from neighboring locations are more likely to meet each other than people from faraway places. Second, public recordkeeping of agents' trading histories is available only within their own location.

We begin by studying conditions that guarantee that trade takes place only among agents from nearby villages and only via the use of credit. In this case, money has no possible welfare-improving role. Monetary trades might emerge only when interactions with faraway villages become sufficiently frequent. In that case, both means of payment coexist. Credit is used in trades among neighbors, whereas money is used in trades among "strangers." 
The idea that credit-like instruments are used in trades among agents with known histories while currency is used in trades among relative strangers is not new. Townsend (1989), for example, studies a model in which both types of transactions coexist. In his model, as in ours, money and credit differ in their communication and recordkeeping aspects. ${ }^{2}$ Our work is most closely related to a number of papers that study credit in a search-based setup starting with Diamond (1990). Kranton (1996) studied the sustainability of reciprocal exchange. Shi (1996) and Corbae and Ritter (1997) study money and credit in a search setup. Unlike them, we do not allow pairs of agents to form ongoing relationships by staying together for more than one period. Finally, Kocherlakota and Wallace (1998) build on Kocherlakota (1998) and study the coexistence of money and credit in an environment where public record-keeping is incomplete because it is updated with a lag.

In our model, record-keeping is incomplete in the sense that it is available only locally. We demonstrate that money is essential in facilitating trade among agents who meet infrequently, and who lack access to each other's trading histories. Our main finding is that when no direct cross-location informational flow is possible, money is useful in providing a partial substitute for it. Thus, while monetary and credit transactions coexist, they differ in their scope. If an agent fails to perform a credit transaction, he loses the opportunity to trade again with the village of the agent that experienced the deviation. On the other hand, if an agent fails to produce in exchange for money, he loses the opportunity to trade with agents from a large number of villages in the near future. Thus, monetary exchange allows for the possibility

\footnotetext{
${ }^{2}$ In earlier work Lucas (1980) suggested that a friction involved in establishing one's creditworthiness might lead to the coexistence of money and credit transactions since, in that case, money will economize on record-keeping costs. More recently, Lacker and Schreft (1996) study a model in which "credit transaction costs," which, unlike the cost of monetary trade, are assumed to increase with distance, give rise to a coexistence of monetary and credit transactions. Our model could be thought of as one providing micro-foundations for such transactions costs.
} 
of a "punishment" that is less severe but broader in scope than denial of future credit. This feature of monetary exchange is shown to partially overcome the lack of a cross-location informational flow. Our model allows us to characterize the fraction of transactions that are monetized as a function of the cross-location informational restrictions.

It is worth mentioning that while here we concentrate on the implications of local matching and record-keeping for monetary theory, our setup might be useful in the study of other issues that involve local interactions. For example, search in labor markets often has a local feature, and reputation of certain goods and services is often available only locally.

The paper proceeds as follows. Section 2 offers an informal description of the model, while Section 3 describes the environment. Section 4 presents the benchmark case of uniform matching. Section 5 contains the main results. Section 6 concludes the paper.

\section{AN INFORMAL DESCRIPTION OF THE MODEL}

As certain aspects of our model are non-standard, we first describe the setup informally. In the standard random matching model, credit cannot exist since agents do not meet repeatedly, and there is no record-keeping of past trades. We amend the standard model in two ways: by assuming that agents meet different sets of agents with different probabilities, and by introducing record-keeping at the local level. Although other interpretations are possible, we interpret the different meeting probabilities as the result of the agents' respective geographic distance from one another. Agents populate villages that are symmetric, each consisting of a large number (formally, a continuum) of agents. Agents are specialized in production and consumption of goods and are assumed to be randomly matched in pairs in each period. Trade is possible in the case where a potential producer meets with someone that likes his 
production good (hence called a single-coincidence meeting). The distribution of all agents' characteristics is the same across villages. With high probability, each agent meets someone from his own village, and the probability of meeting another agent decreases as the distance from the village that the agent belongs to increases. In addition, we assume that each individual's trading history is public information only within the village where they belong, and only in regard to their meetings with agents from that village. ${ }^{3}$

What are the likely methods of payment in this environment? One possibility is that producers offer to produce without requiring immediate payment regardless of the consumer's location of origin. In the context of our model, we will identify such a non-monetized exchange with a credit transaction. Suppose that if one agent deviates from this implicit agreement, say by refusing to produce for someone from village $j$, the deviation triggers a collective punishment to permanent no-trade between the deviator and village $j$. One may think that this threat might induce an equilibrium in which credit is used in all trades. However, consider an agent in a meeting with someone from a very "faraway" village. The potential producer has a strong incentive to deviate. The disutility of producing is suffered now, while the punishment will be borne sometime in the distant future. ${ }^{4}$ Since the future is discounted, the producer might refuse to produce. Our main result establishes conditions for the existence of a critical distance (frequency of meetings) such that in a stationary equilibrium, only credit transactions take place among close neighbors (people whose frequency of

\footnotetext{
${ }^{3}$ As we mentioned earlier, geographic distance is one possible reason why matching and recordkeeping are local, and other interpretations of the model are, indeed, possible. For example, one could argue that academic economists are "close" to each other in the sense that they meet frequently and share information, say in conferences, regardless of their actual geographic location. Our results would apply under such alternative interpretations of the "distance" function.

${ }^{4}$ This requires that the close neighbors of the deviating producer remain unaware of his deviation. Later we make assumptions that guarantee that this will be true in our setup.
} 
meetings is higher than a critical value), while only monetary transactions occur in meetings between people from faraway places (people whose frequency of meetings is lower than the critical value).

\section{THE ENVIRONMENT}

Time is discrete and the horizon is infinite. The economy is populated by a continuum of agents of total measure 1. Agents are matched bilaterally in every period. There is an (odd) number of villages denoted by $j=1,2, \ldots, n$, forming a partition of the unit circle with a continuum of agents in each village. There is a finite number, $k \geq 3$, of perishable and indivisible consumption goods. Agents specialize in consumption and production in a symmetric fashion. At the beginning of each period, there is a $\left[0, \frac{1}{k}\right]$ continuum of each type. Individuals of type $i$ consume good $i$ and produce good $i+1$ (modulo $k$ ), where $i=0, \ldots, k-1$. The instantaneous utility from consumption is $u>0$. Production requires an effort that gives disutility $e>0$, where $u>e$. Agents maximize expected discounted utility, and the time discount factor is $\beta \in(0,1)$. Because $k \geq 3$, there is no double coincidence of wants. We assume that a fraction $M$ of agents each start with one unit of indivisible, storable, and intrinsically useless fiat money. Current period money holdings are observable within a match. We impose an upper bound on individual holdings; i.e., individuals can hold at most one unit of currency or one unit of a good. This implies that, in meetings where there is monetary trade, one unit of money is exchanged for one unit of good. ${ }^{5}$ Agents are assigned to villages at the beginning of time. The initial distribution of all agents' characteristics, including money holdings, is uniform across consumption types and villages. We will concentrate on stationary equilibria that respect this symmetry.

\footnotetext{
${ }^{5}$ The indivisibility assumption is largely for simplicity. Introducing endogenous prices as in Shi (1995) and Trejos and Wright (1995) would complicate our analysis without qualitatively changing the results.
} 
One difference between the environment of our model and the standard setup in existing search models is in the matching technology. Matching here is not uniform but local. Each agent is matched with an agent from his own village with probability $p_{0}$; he is matched with an agent from either of the two immediately neighboring villages with probability $p_{1}$, where $p_{0}>p_{1}>p_{2}>\ldots$, etc. There is no loss of generality in concentrating our analysis on a distinguished agent from a generic village, $j$. Denote by $d$ the distance between our generic village and any other village, $j^{\prime}$; i.e., $d=\left|j-j^{\prime}\right|$. Hence, $p_{d}$ stands for the probability that our distinguished agent is matched with an agent from a $d$-order neighboring village, where $\sum_{d=0}^{\frac{n-1}{2}} p_{d}=1$. Agents return to their village at the end of each period. We assume that the random matching technology operates in two levels. First, in any given period each village is randomly assigned to one and only one village (possibly to itself). Then, agents from the matched villages are randomly matched in pairs. The role of this assumption is explained later.

Another novel feature of our model is that agents from different villages cannot exchange information regarding trading histories. This captures the feature that, especially in early village economies, it was costly to have access to credit histories, and these costs increased as a function of distance. A potential deviation experienced by an agent from village $j$ is automatically communicated to all agents in village $j$ by the agent that experienced the deviation and triggers a punishment to permanent no trade with the deviating agent by all agents in village $j .{ }^{6}$ Of course, the deviator has no incentive to reveal his deviation. Also, actions during meetings with agents

\footnotetext{
${ }^{6}$ Following common practice, we assume that a deviation triggers a punishment to the worst "reasonable" equilibrium. In general, a lesser punishment requires a higher discount factor in order to maintain the same composition of credit and monetary trades. In a different context, Cole and Kehoe (1995) study a model of sovereign debt and demonstrate that multiple relationships and spillovers across them can support debt through the threat of similar collective punishments.
} 
from other villages are not publicly observable in the deviator's own village. The assumption that agents from one village can meet with agents from only one other village within a period ensures two features that are important for what follows: (1) information about trading histories remains local and (2) there is no aggregate uncertainty so that the model remains tractable. ${ }^{7}$

When two agents have a single-coincidence meeting, they simultaneously make a trading proposal after they observe each other's type, current money holdings, and village of origin. The potential consumer has three choices: (1) to request production without payment, (2) to offer money in exchange for production, and (3) to propose no trade. The producer also has three choices: (1) to offer to produce requesting no payment, (2) to request money in exchange for production, and (3) to propose no trade. If the two proposals match, the resulting trades are realized. If they are different, then a punishment is triggered. ${ }^{8}$

Consider our distinguished agent in a meeting with an agent from a village that is $d$ steps away. We denote by $\alpha^{d}$ the probability with which an agent requests money in exchange for production, and by $m^{d}$ the probability that he offers money in order to consume. Similarly, let $g_{M_{p}, M_{c}}^{d}$ and $h_{M_{p}, M_{c}}^{d}$ be the respective probabilities that an agent produces requesting no payment and that he requests production offering no payment when the producer's and the consumer's money holdings are given by $M_{p}$ and $M_{c}$, respectively. The remaining probability is assigned to the event that a no trade proposal is made.

Of course, the punishment to permanent no-trade is arbitrary, and one could think of several alternatives. The harshest possible punishment that a deviated-upon village

\footnotetext{
${ }^{7}$ Without this restriction in the matching technology, positive fractions of agents from any village would meet with agents from all other villages. Thus, if one village is informed of a deviation and triggers global autarky, this could lead to global autarky two periods after the deviation occurred.

${ }^{8}$ We ignore the case where the producer offers to produce requesting no payment while the consumer offers money. This, of course, would never occur in equilibrium.
} 
could adopt is to revert to permanent autarky during all future meetings with all villages. ${ }^{9}$ Even if this punishment is adopted, a long lag before it reaches the deviator guarantees that the qualitative properties of our results still hold. On the other hand, we could adopt the less harsh punishment of allowing monetary exchanges in future meetings with a known deviator. ${ }^{10}$ As we mentioned earlier, this would complicate the analysis without qualitatively changing the results.

We now describe the general value functions, special cases of which we use later. A symmetric equilibrium consists of values for $g_{M_{p}, M_{c}}^{d}, h_{M_{p}, M_{c}}^{d}, \alpha^{d}$ and $m^{d}$ that satisfy the two value functions below, and are such that $g_{M_{p}, M_{c}}^{d}=h_{M_{p}, M_{c}}^{d}$ and $\alpha^{d}=m^{d}$. We concentrate on the symmetric steady state value function of an agent from a generic village $j$. We let $V_{0}$ and $V_{1}$ denote the value function of an agent with and without money, respectively. For the case of an agent with no money, we have

$$
\begin{aligned}
& V_{0}=\sum_{d=0}^{\frac{n-1}{2}} p_{d} \\
& \left\{\begin{array}{c}
\left(1-\frac{2}{k}\right) \beta V_{0}+\frac{1}{k}(1-M)\left[\max _{g_{00}^{d}}\left(-g_{00}^{d} h_{00}^{d} e+\beta V_{0}\right)+\max _{h_{00}^{d}}\left(g_{00}^{d} h_{00}^{d} u+\beta V_{0}\right)\right] \\
\left(1-m^{d}\right)\left(-g_{01}^{d} h_{01}^{d} e+\beta V_{0}\right) \\
+\frac{1}{k} M\left\{\max _{g_{01}^{d}}\left\{\begin{array}{c}
\left(1-\beta V_{1}\right)+g_{01}^{d}\left(-e+\beta V_{0}\right) \\
+m^{d}\left\{\max _{\alpha^{d}}\left[\begin{array}{c}
\alpha^{d}(-e+\beta \\
+\left(1-\alpha^{d}-g_{01}^{d}\right) \beta V_{0}
\end{array}\right]\right\} \\
+\max _{h_{10}^{d}}\left(g_{10}^{d} h_{10}^{d} u+\beta V_{0}\right)
\end{array}\right\}\right.
\end{array}\right\}
\end{aligned}
$$

The first part of the value function describes the case of a meeting in which there is

\footnotetext{
${ }^{9}$ This punishment defines one end of the spectrum, but it is unattractive. For example, agents in the deviated-upon village know the location of the deviator. It does not seem reasonable to punish themselves by adopting autarky in meetings within the village, or in meetings with their close neighbors, especially if the deviating agent's village is "far away."

${ }^{10}$ This is especially relevant since both the deviator and his partner might have an incentive to perform a monetary trade instead of making no proposals. However, notice that, given our mechanism, making no proposals in such a meeting remains an equilibrium. In fact, if we assume an arbitrarily small cost of making a proposal, it is a strict equilibrium.
} 
no coincidence of wants. The second part describes the case of a single coincidence meeting as a producer and as a consumer, respectively, with an agent that has no money holdings. In that case, our distinguished agent chooses whether to offer credit and whether to receive credit, respectively. Finally, the third part describes the case of a single coincidence meeting as a producer and as a consumer, respectively, with an agent that holds money. In that case, our distinguished agent chooses whether to offer credit or to offer to produce in exchange for his partner's money holdings, etc. Similarly, for the case of a distinguished agent with money, we have

$$
\begin{aligned}
& V_{1}=\sum_{d=0}^{\frac{n-1}{2}} p_{d} \\
& \left\{\begin{array}{c}
\left(1-\frac{2}{k}\right) \beta V_{1}+\frac{1}{k} M\left[\max _{g_{11}^{d}}\left(-g_{11}^{d} h_{11}^{d} e+\beta V_{1}\right)+\max _{h_{11}^{d}}\left(g_{11}^{d} h_{11}^{d} u+\beta V_{1}\right)\right] \\
\max _{g_{10}^{d}}\left(-g_{10}^{d} h_{10}^{d} e+\beta V_{1}\right) \\
+\max _{m^{d}}\left\{\begin{array}{c}
\left(1-m^{d}\right)\left[\max _{h_{01}^{d}}\left(g_{01}^{d} h_{01}^{d} u+\beta V_{1}\right)\right] \\
+m^{d}\left[\begin{array}{c}
\alpha^{d}\left(u+\beta V_{0}\right)+g_{01}^{d}\left(u+\beta V_{1}\right) \\
+\left(1-\alpha^{d}-g_{01}^{d}\right) \beta V_{1}
\end{array}\right]
\end{array}\right\}
\end{array}\right\} .
\end{aligned}
$$

\section{A BENCHMARK CASE - UNIFORM MATCHING}

Our main focus is the study of a model where both record-keeping and matching are local. In order to separate the role of these two assumptions, we first consider the case where matching is uniform, while information about histories is available only at the local level. Suppose there are $n$ villages and $p_{d}=p=\frac{1}{n}$, for all $d$. The following proposition asserts that in this environment, money and credit payments cannot coexist in a non-trivial way. More precisely, credit exchange across all meetings can be supported as an equilibrium outcome for low values of $n$, while credit exchange cannot be supported for high values of $n$.

Proposition 1 Suppose that $\beta \in(0,1)$. There is a unique $n^{*}$ such that if $p \in\left(\frac{1}{n^{*}}, 1\right)$, 
there exists a pure credit equilibrium, and money is not essential. If $p \in\left(0, \frac{1}{n^{*}}\right)$, there exists no equilibrium with credit transactions.

The above follows as a Corollary of the main Proposition in the next section. The intuition is simple. A deviation from offering credit triggers permanent no-trade with the village of the deviated-upon agent. Producing implies a certain amount of disutility that the producer suffers today. On the other hand, the potential cost from the autarky punishment depends on the frequency with which the penalty is experienced in the future. If the probability of meeting the same village is high enough, the best response is to offer credit. Otherwise, the best response will be to deviate. Since the meeting probabilities are equal, the result follows. ${ }^{11}$

\section{MONEY AND CREDIT UNDER LOCAL MATCHING}

In this section we derive our main proposition establishing conditions that guarantee the coexistence of monetary and credit transactions. Recall that the random matching technology operates in two steps. First, a village is matched to any other, including itself, with some probability. Then individual agent matches occur. We first prove some preliminary results. The first Lemma asserts that the search economy in Kocherlakota (1998) follows as a special case of our setup if we shut down all meetings across different villages $\left(p_{0}=1\right)$. In that case, each village is an isolated economy under uniform matching, and a high discount factor guarantees the existence of a credit equilibrium. No monetary arrangement can result in a higher

\footnotetext{
${ }^{11}$ In our model the severity of the punishment increases as the expected frequency of meetings between two locations increases. What is important in general is that the expected value of the potential punishment is high. This could be accomplished in other ways. For example, assuming that matching probabilities are equal but that agents derive lower utility from consuming goods produced by "far away" agents would create the same effect. We thank an anonymous referee for suggesting this alternative.
} 
frequency of consumption than the credit equilibrium. This is because in a credit equilibrium trade takes place in each single-coincidence meeting, while with money it is also needed that in at least some meetings the consumer has money and the producer does not. We will concentrate on the Pareto superior credit regime whenever such a regime can be supported.

Lemma 1 Suppose that $p_{0}=1$. There exist $\beta^{C}$ and $\beta^{M}$ with $0<\beta^{C}<\beta^{M}<1$ such that for all $\beta \in\left(\beta^{C}, 1\right)$, a credit equilibrium exists, and for all $\beta \in\left(\beta^{M}, 1\right)$, a monetary equilibrium exists. Welfare in the credit equilibrium is strictly higher than in any equilibrium in which money is used.

Proof: We let $V^{C}$ be the value function under a credit regime, and we denote by $V_{1}^{M}$ and $V_{0}^{M}$ the value functions of a money holding and a non-money holding agent, respectively, under a monetary regime. In a credit equilibrium we have that $V^{C}=$ $\frac{u-e}{k(1-\beta)}>0$. In order for $g^{d}=1$ to be the best response for a producer in a single coincidence meeting where $h^{d}=1$, we need that $-e+\beta V^{C} \geq 0$, which implies that $\beta \geq \frac{e k}{e k+u-e} \equiv \beta^{C}$. On the other hand, since $u+\beta V^{C}>0$, we have that $h^{d}=1$ is the best response given $g^{d}=1$. That is, the credit equilibrium exists provided that $\beta \in\left(\beta^{C}, 1\right)$. In a monetary equilibrium we have

$$
\begin{aligned}
V_{1}^{M} & =\frac{1}{k}(1-M)\left(u+\beta V_{0}^{M}\right)+\left[1-\frac{1}{k}(1-M)\right] \beta V_{1}^{M} \\
V_{0}^{M} & =\frac{1}{k} M\left(-e+\beta V_{1}^{M}\right)+\left(1-\frac{1}{k} M\right) \beta V_{0}^{M} .
\end{aligned}
$$

For a monetary equilibrium to exist, we need that $-e+\beta V_{1}^{M} \geq \beta V_{0}^{M}$. Solving for $V_{0}^{M}$ and $V_{1}^{M}$ and substituting, we have that the inequality holds if $\beta \geq \frac{e k}{e k+(u-e)(1-M)} \equiv$ $\beta^{M}$. Average welfare in the monetary equilibrium is given by $W^{M}=(1-M) V_{0}^{M}+$ $M V_{1}^{M}$. It is easy to see that $W^{M}$ reaches the maximum, $\bar{W}^{M}$, when $M=\frac{1}{2}$. In that case, $\bar{W}^{M}=\frac{u-e}{4 k(1-\beta)}$, which is lower than average welfare in the credit equilibrium. 
The next Lemma asserts that the welfare cost from not being in a credit regime increases in the expected frequency of meetings between two villages. In addition, a sufficient condition is derived for credit transactions to be sustainable. Intuitively, autarky implies an overall lower expected frequency of consumption. Thus, the higher the frequency of meetings between two villages, the bigger the cost of reverting to no trade. Since punishment becomes more painful, it is easier to support the credit regime. Define $\bar{p}_{d} \equiv \frac{e k(1-\beta)}{\beta(u-e)}$.

Lemma 2 Suppose that $\beta$ is sufficiently high. Consider two villages that are matched with probability $p_{d}$. The difference between the value functions under credit and under autarky between them increases in $p_{d}$. Furthermore, if $p_{d} \in\left(\bar{p}_{d}, 1\right)$, all transactions between the two villages use credit, while if $p_{d} \in\left(0, \bar{p}_{d}\right)$, there is no equilibrium with credit transactions between the two.

Proof: Consider a distinguished agent from a generic village, $j$. Let $p^{C}\left(p^{M}\right)$ be the sum of the matching probabilities with the villages that use credit (money) in meetings with agents from $j$. Let $p^{A}$ be the sum of the matching probabilities of the villages that are in autarky with our distinguished agent. Let $V_{0}^{C}, V_{1}^{C}, V_{0}^{A}$, and $V_{1}^{A}$ stand for the value functions under a credit regime and under autarky between our distinguished agent and an arbitrary village $j^{\prime}$. The value functions of our distinguished agent are given by

$$
\begin{aligned}
V_{0}^{C}= & \left(p^{C}+p_{d}\right)\left[\beta V_{0}^{C}+\frac{1}{k}(u-e)\right] \\
& +p^{M}\left[\left(1-\frac{1}{k} M\right) \beta V_{0}^{C}+\frac{1}{k} M\left(\beta V_{1}^{C}-e\right)\right]+p^{A} \beta V_{0}^{C}, \\
V_{1}^{C}= & \left(p^{C}+p_{d}\right)\left[\beta V_{1}^{C}+\frac{1}{k}(u-e)\right] \\
& +p^{M}\left\{\left[1-\frac{1}{k}(1-M)\right] \beta V_{1}^{C}+\frac{1}{k}(1-M)\left(u+\beta V_{0}^{C}\right)\right\}+p^{A} \beta V_{1}^{C},
\end{aligned}
$$


and

$$
\begin{aligned}
V_{0}^{A}= & p^{C}\left[\beta V_{0}^{A}+\frac{1}{k}(u-e)\right] \\
& +p^{M}\left[\left(1-\frac{1}{k} M\right) \beta V_{0}^{A}+\frac{1}{k} M\left(\beta V_{1}^{A}-e\right)\right]+\left(p^{A}+p_{d}\right) \beta V_{0}^{A}, \\
V_{1}^{A}= & p^{C}\left[\beta V_{1}^{A}+\frac{1}{k}(u-e)\right] \\
& +p^{M}\left\{\begin{array}{r}
{\left[1-\frac{1}{k}(1-M)\right] \beta V_{1}^{A}} \\
+\frac{1}{k}(1-M)\left(u+\beta V_{0}^{A}\right)
\end{array}\right\}+\left(p^{A}+p_{d}\right) \beta V_{1}^{A} .
\end{aligned}
$$

In order to support credit transactions between $j$ and $j^{\prime}$, we need that $-e+\beta V_{0}^{C} \geq$ $\beta V_{0}^{A}$ and $-e+\beta V_{1}^{C} \geq \beta V_{1}^{A}$. It is easy to check that

$$
V_{1}^{C}-V_{1}^{A}=V_{0}^{C}-V_{0}^{A}=V^{C}-V^{A}=\frac{p_{d}(u-e)}{k(1-\beta)},
$$

which is increasing in $p_{d}$. The expressions for $V_{0}^{C}-V_{0}^{A}$ and $V_{1}^{C}-V_{1}^{A}$ are independent of how agents in village $j$ trade in meetings with agents from villages other than $j^{\prime}$. Using the first part and substituting $\frac{p_{d}(u-e)}{k(1-\beta)}$ for the difference $V^{C}-V^{A}$, we have that the incentive conditions are satisfied if $p_{d} \geq \frac{e k(1-\beta)}{\beta(u-e)} \equiv \bar{p}_{d}$.

Notice that $\bar{p}_{d}$ is decreasing in $\beta$. It equals zero when $\beta=1$ and equals one when $\beta=\beta^{C}$. It is easy to show that $\bar{p}_{d}$ increases in $k$ and $e$, while it decreases in $u$ and $\beta$. As the probability of single coincidence becomes smaller, the welfare under a credit regime is reduced. Thus, it becomes harder to support the credit equilibrium. A higher production cost or a lower utility from consumption also makes the credit equilibrium harder to support by reducing the value of consumption. On the other hand, a higher discount factor helps to lower the critical probability.

The previous Lemma does not imply the existence of monetary trades. Producers in meetings where credit cannot be supported will produce in exchange for money provided that acquiring money sufficiently increases their probability of future consumption. This requires that the producers will be able to use their earned money in 
the near future which, in turn, requires that a meeting with someone from a distant village is likely.

The next Lemma gives sufficient conditions for all transactions in meetings between agents from two villages to be monetary. Let $B$ be the set of villages that are each matched to our generic village, $j$, with probability less than $\bar{p}_{d}$, so that a credit arrangement cannot be supported in meetings between agents from $j$ and any of the villages in $B$. Let $p_{B}$ be the probability that village $j$ is matched to a village in $B$. That is, $p_{B}=\sum_{d=0}^{\frac{n-1}{2}} p_{d}$, for $p_{d}<\bar{p}_{d}$. Define $\bar{p}_{B} \equiv \frac{e k(1-\beta)}{\beta(1-M)(u-e)}$.

Lemma 3 Consider two villages that are matched with probability lower than $\bar{p}_{d}$. Provided that $p_{B}>\bar{p}_{B}$, there exists an equilibrium where all transactions between the two villages are monetary.

Proof: For $\beta$ sufficiently high, a credit equilibrium can be supported in meetings between agents from village $j$ and any village in the complement of $B$. The value functions for our distinguished agent can be written as

$$
\begin{aligned}
V_{1}= & \left(1-p_{B}\right)\left[\beta V_{1}+\frac{1}{k}(u-e)\right] \\
& +p_{B}\left\{\frac{1}{k}(1-M)\left(u+\beta V_{0}\right)+\left[1-\frac{1}{k}(1-M)\right] \beta V_{1}\right\} \\
V_{0}= & \left(1-p_{B}\right)\left[\beta V_{0}+\frac{1}{k}(u-e)\right] \\
& +p_{B}\left[\frac{1}{k} M\left(-e+\beta V_{1}\right)+\left(1-\frac{1}{k} M\right) \beta V_{0}\right] .
\end{aligned}
$$

For a monetary equilibrium to exist in a meeting between agents from village $j$ and a village $j^{\prime} \in B$, it is sufficient that $-e+\beta V_{1}>0+\beta V_{0}$. Solving for $V_{0}$ and $V_{1}$ and substituting, we find that the inequality holds if $p_{B} \geq \frac{e k(1-\beta)}{\beta(1-M)(u-e)} \equiv \bar{p}_{B}$.

We are now ready to state our main Proposition. A credit regime between two villages can be supported if and only if the probability of a meeting between them is 
greater than $\bar{p}_{d}$. In the case where credit is not sustainable, monetary trades may be supported if acquiring money sufficiently increases the probability of consuming in the future; i.e., if a meeting with some other "faraway" village in the near future is likely. To better understand the economic principle at work, consider a community of people that trade almost exclusively among themselves via the use of credit. Suppose that, in a very unlikely event, one of the residents meets a consumer from far away. It will certainly not be individually optimal to produce for free for the stranger, but what if the stranger offers money in exchange for production? Will the potential producer accept the money and produce? If future meetings are expected to be only with residents of the community, money will be of no use for a long time and will be rejected. On the other hand, if meetings with strangers are frequent enough, the producer can use money to finance consumption in the near future, and so he will accept it. These observations define the existence of a critical probability that separates the two types of transactions.

Proposition 2 Suppose that $\beta \in\left(\beta^{C}, 1\right)$. Transactions between village $j$ and villages that are matched to $j$ with probability $p_{d} \in\left(\bar{p}_{d}, 1\right)$ use credit and, provided that the probability of a meeting between $j$ and a village in $B$ is greater than $\bar{p}_{B}$, transactions between $j$ and villages that are matched to $j$ with probability $p_{d} \in\left(0, \bar{p}_{d}\right)$ are monetary.

Proof: The sufficient conditions of $\beta$ to support the credit regime and monetary regime are given in our earlier discussion related to Lemma 1 . The existence of a credit regime when $p_{d} \in\left(\bar{p}_{d}, 1\right)$ and the non-existence of a credit regime when $p_{d} \in\left(0, \bar{p}_{d}\right)$ follows from Lemma 2. The existence of a monetary regime when $p_{d} \in\left(0, \bar{p}_{d}\right)$ and $p_{B} \in\left(\bar{p}_{B}, 1\right)$ follows from Lemma 3.

To illustrate this proposition, we present a simple example. Let $u=8, e=2, k=3$, and $M=0.5$. Then, $\bar{p}_{d}=\frac{1-\beta}{\beta}$ and $\bar{p}_{B}=\frac{2(1-\beta)}{\beta}$. These are plotted in Figure 1 . The 


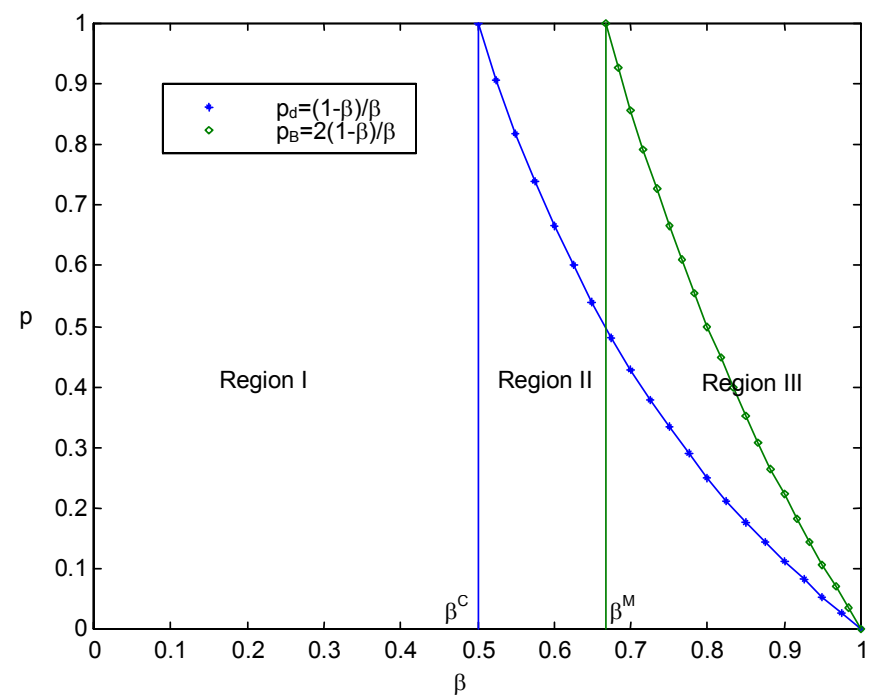

FIG. 1. Regions for $p_{d}, p_{B}, \beta$ and Equilibria

Figure also demonstrates the regions of values for $p_{d}, p_{B}$, and $\beta$, and the equilibria that are supported in these regions. We know that $\bar{p}_{d}=1$ implies that $\beta=\beta^{C}$ and that $\bar{p}_{B}=1$ implies that $\beta=\beta^{M}$. The two vertical lines in Figure 1 give rise to three regions. Consider the generic village $j$ matched with village $j^{\prime}$ with probability $p_{d}$. In region I, autarky is the only equilibrium since the discount factor is too low to support either credit or monetary transactions between the two villages. In region II, if in addition $p_{d}$ lies above $\bar{p}_{d}$, all transactions between $j$ and $j^{\prime}$ use credit. If $p_{d}$ lies below, no trade takes place. Monetary transactions cannot be supported in this region since $p_{B}$ is always less than $\bar{p}_{B}$ ( $\beta$ is always less than $\left.\beta^{M}\right)$. In region III, if in addition $p_{d}$ lies above $\bar{p}_{d}$, all transactions between $j$ and $j^{\prime}$ use credit. If $p_{d}$ lies below, there are two possibilities: autarky or monetary exchange depending on how many other villages lie below $\bar{p}_{d}$. If $p_{B}$ is above $\bar{p}_{B}$, transactions between $j$ and $j^{\prime}$ are monetary; otherwise autarky prevails. Figure 2 gives the same information regarding region III, in the $\left(p_{d}, p_{B}\right)$ space. 


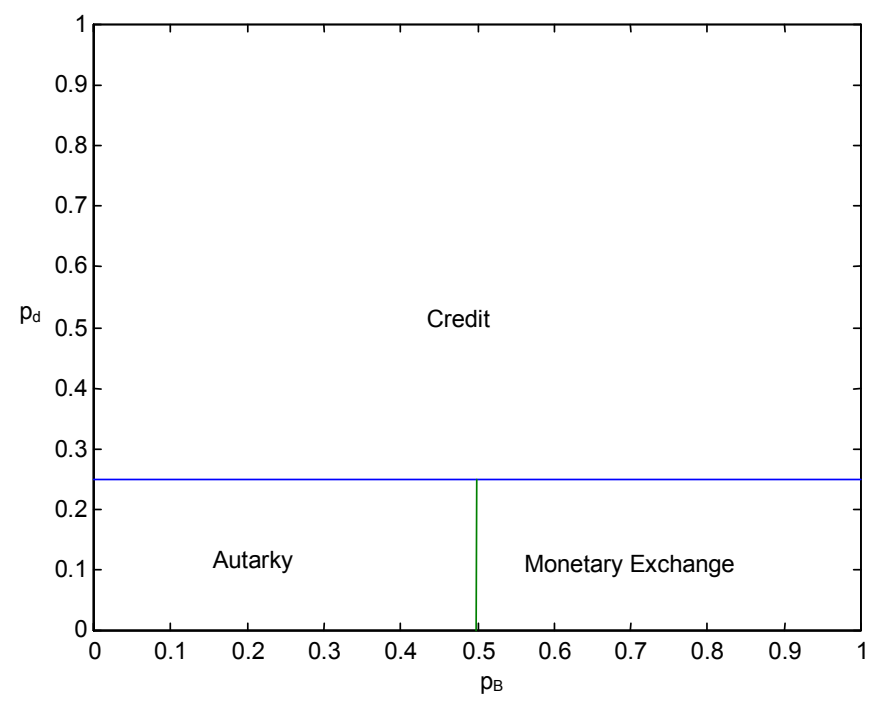

FIG. 2. Regions for $p_{d}, p_{B}$ and Equilibria, $\beta=0.8$

\section{CONCLUSIONS}

We studied conditions leading to an equilibrium in which credit is used among agents that meet each other frequently, while money is used in transactions among those that meet only infrequently. Our main finding is that when no direct crosslocation informational flow is possible, money provides a partial substitute for it. Thus, monetary and credit transactions coexist but differ in their scope.

It is hard to know the exact form of non-monetized transactions in early economies, but observations of contemporary village economies provide some clues. In their study of the financial structure of three villages in India, Lim and Townsend (1998) found evidence consistent with credit functioning well within villages, while, at the same time, external credit markets do not function as well, possibly due to a disadvantage in information sharing. Perhaps the most appropriate interpretation of our model is as a parable of a medieval village economy. Our main proposition suggests that 
money might "emerge" when, as a result of increased mobility, trades among people from faraway locations become sufficiently frequent.

An important question concerns the general class of lattices that give rise to results similar to ours. Indeed, if the location interpretation of the matching probabilities is dropped in favor of a more general one, the assumption that agents are located on a circle, or a torus, appears overly restrictive. We conjecture that versions of our results will hold in more general lattices provided that there is a lag between the time when a deviation occurs and when the resulting punishment reaches the deviator. A formal study of this question, however, is beyond the scope of this paper.

In future work we plan to introduce multiple locally-issued fiat objects and study their circulation and redemption properties as a function of the local matching rule. It would also be interesting to study monetary injections and price dispersion in the context of a local interaction model with endogenous prices. In such a model, newly injected money is likely to stay within a small set of locations in the short run. In addition, locally issued money could circulate globally, but at a discount that is proportional to the respective distance from the village of issuance.

\section{REFERENCES}

Cole H., and P. Kehoe (1995): The Role of Institutions in Reputation Models of Sovereign Debt. Journal of Monetary Economics 35(1), 45-64.

Corbae D., and J. Ritter (1997): Money and Search with Enduring Relationships, Manuscript.

Diamond P. (1990): Pairwise Credit in a Search Equilibrium. Quarterly Journal of Economics 105, 285-320.

Kiyotaki N., and R. Wright (1989): On Money as a Medium of Exchange. Journal of Political Economy 97(4), 927-954.

Kocherlakota N. (1998): Money is Memory. Journal of Economic Theory 81(2), 
$232-251$.

Kocherlakota N., and N. Wallace (1998): Incomplete Record-Keeping and Optimal Payment Arrangements. Journal of Economic Theory, 81(2) 272-289.

Kranton R. (1996): Reciprocal Exchange: A Self-Sustaining System. American Economic Review 86(4), 830-851.

Lacker J. and S. Schreft (1996): Money and Credit as Means of Payment. Journal of Monetary Economics 38, 3-23.

Lim Y., and R. Townsend (1998): General Equilibrium Models of Financial Systems: Theory and Measurement in Village Economies. Review of Economic Dynamics 1(1), $59-118$.

Lucas R.E. Jr. (1980): Equilibrium in a Pure Currency Economy. Economic Inquiry 18(2), 203-220.

Shi S. (1995): Money and Prices: A Model of Search and Bargaining. Journal of Economic Theory 67, 467-496.

Shi S. (1996): Credit and Money in a Search Model with Divisible Commodities. Review of Economic Studies 63, 627-652.

Townsend R.M. (1989): Currency and Credit in a Private Information Economy. Journal of Political Economy 97(6), 1323-1344.

Trejos A., and R. Wright (1995): Search, Bargaining, Money and Prices. Journal of Political Economy 103, 118-141. 